\title{
Journal of Bone and Mineral Metabolism Best Paper Award 2018
}

○ The Japanese Society for Bone and Mineral Research and Springer Japan KK, part of Springer Nature 2018

The Journal of Bone and Mineral Metabolism Best Paper Award was established in 2008. Candidates for the award must be members of the Japanese Society of Bone and Mineral Research, and the winner is honored at the Society's Annual Meeting.

We are pleased to announce that the following article has received JBMM Best Paper Award.

\section{"Osteoporosis is highly prevalent in Japanese males with chronic obstructive pulmonary disease and is associated with deteriorated pulmonary function" by}

Reiko Watanabe, Takeshi Tanaka, Keisuke Aita, Masaaki Hagiya, Toshiaki Homma, Kyoko Yokosuka, Hisami Yamakawa, Tsutomu Yarita, Nobuyuki Tai, Junko Hirano, Daisuke Inoue, Ryo Okazaki

J Bone Miner Metab (2015) 33:392-400

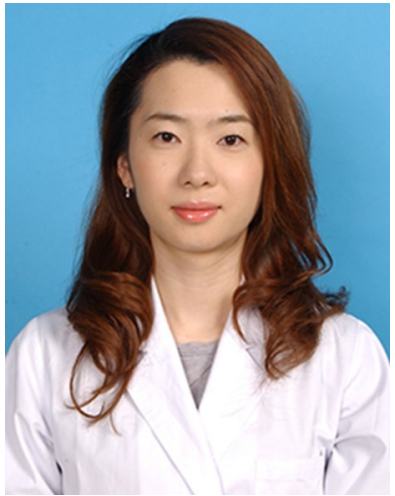

Dr. Reiko Watanabe

\begin{abstract}
Osteoporosis has recently been recognized as a major comorbidity in chronic obstructive pulmonary disease(COPD). We conducted a cross-sectional study in a cohort of 136 Japanese males with COPD to evaluate the prevalence of vertebral fracture (VF) and to explore its relationship with pulmonary function parameters. VFs were present in 108 (79.4\%); multiple and severe (SQ grade 2 or 3) VFs were found in 77 (56.6\%) and 25 (18.4\%), respectively. Multivariate logistic regression analyses revealed that decrease in forced expiratory volume in one second (FEV1.0)/forced vital capacity (FVC) [odds ratio (OR) 0.963, 95\% confidence interval (CI) 0.929-998, $\mathrm{p}=0.036$ ] was associated with the presence of VF after adjustment for age and that FVC (OR 0.462, 95\% CI 0.220-0.968, p=0.041) and current smoking (OR 2.992, 95\% CI 1.128-7.940, p=0.028) were associated with VF severity (grade 2-3 vs. 1). We also found that FEV1.0 was the sole independent determinant of the number of VFs by stepwise multivariate linear regression ( $\mathrm{p} \backslash 0.001$ ). Bone mineral density (BMD) values were available in 49 subjects. Mean T scores were $-2.0 \pm 1.2$ in femoral neck, $-1.4 \pm 1.2$ in total hip and $-1.1 \pm 1.4$ in lumbar spine. Nineteen patients $(38.8 \%)$ had a BMD T score less than - 2.5. BMD Z scores of all the sites showed a progressive decrease as GOLD stage of COPD advanced (p\0.05). Our results indicate a high prevalence of osteoporosis in Japanese male COPD patients and a strong inter-relationship between the two diseases, reemphasizing the urgent need for appropriate intervention to maintain both bone and lung health.
\end{abstract}

We offer our sincere congratulations on behalf of the Journal of Bone and Mineral M1etabolism, with best wishes for further development of the author's research.

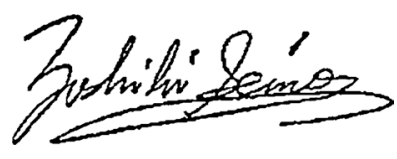

Yoshiki Seino

Editor-in-Chief

Journal of Bone and Mineral Metabolism 